\title{
Convenient Synthesis of Benzo[b]thiophene-5,6-dicarboximide Derivatives and Their Photophysical Properties
}

\author{
Naoki Kobayashi ${ }^{1}$, Shinya Yamamoto $^{1}$, Haruki Shimosasa ${ }^{1}$, Mitsunori Oda $^{1,}{ }^{*}$, Ryuta Miyatake ${ }^{2}$ \\ ${ }^{1}$ Department of Chemistry, Faculty of Science, Shinshu University, Nagano, Japan \\ ${ }^{2}$ Centre for Environmental Conservation and Research Safety, University of Toyama, Toyama, Japan
}

Email address:

mituoda@shinshu-u.ac.jp (M. Oda)

${ }^{*}$ Corresponding author

\section{To cite this article:}

Naoki Kobayashi, Shinya Yamamoto, Haruki Shimosasa, Mitsunori Oda, Ryuta Miyatake. Convenient Synthesis of Benzo[b]thiophene-5,6-dicarboximide Derivatives and Their Photophysical Properties. Modern Chemistry. Vol. 4, No. 5, 2016 , pp. 45-51. doi: $10.11648 /$ j.mc.20160405.11

Received: October 5, 2016; Accepted: October 18, 2016; Published: November 10, 2016

\begin{abstract}
Phosphine-assisted annulation of thiophene-2,3-dicarbaldehyde with $N$-substituted maleimides provided the $N$-substituted benzo[b]thiophene-5,6-dicarboximides in good to high yields. Introduction of cyano and aryl groups to the thiophene moiety of the $N$-cyclohexyl product was achieved by metal-catalyzed coupling reactions via its bromo derivative. Photophysical properties of the products were also reported.
\end{abstract}

Keywords: Annulation, Phosphine, Copper-Mediated Cyanation, Mizoroki-Heck Reaction, Emission

\section{Introduction}

Arene-dicarboximides display unique photophysical and electrochemical properties and have been attractive to be used as organic electronic materials, such as light-emitting diodes, [1] semiconductors, [2] bio-sensors, [3-4] and electron acceptors in solar cells. [5-6] Thus, synthetic improvements to access to these imides have continuously made. [7-8]

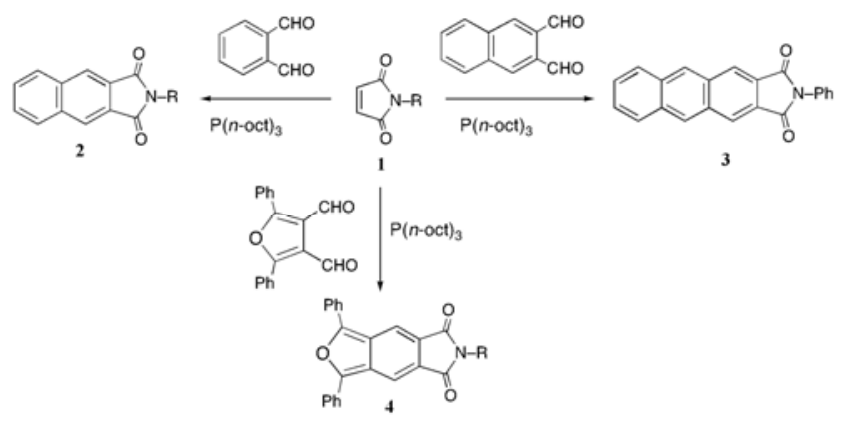

Figure 1. Previously reported annulations by us.

We recently developed an efficient method of annulation between arene-1,2-dicarbaldehydes and maleimides [9-11] by improving the previously reported Haddadin's result. [12] various naphthalene-, anthracene-, and isobenzofuran-dicarboximides $2-4$ can be obtained in one-pot from the corresponding arene-1,2-dicarbaldehydes and maleimides in good to high yields (Fig. 1). These reactions proceed efficiently with the aid of trialkylphosphine in refluxing dioxane. It is worthy to note that the reaction procedure of this method is very simple, because the products were obtained directly from the reaction mixtures just by filtration.

In this paper, we present the synthesis of the benzo[b]thiophene-5,6-dicarboximides by applying this method to annulation of thiophene-2,3-dicarbaldehyde with $N$-substituted maleimides (Fig. 2), derivatization of the $\mathrm{N}$-cyclohexyl product, and also photophysical properties of the products.

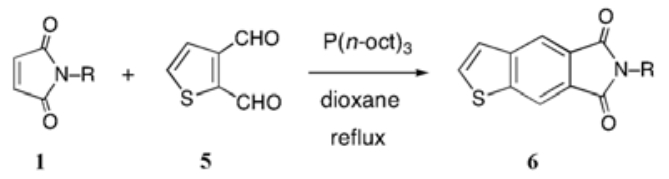

Figure 2. A synthetic route to $N$-substituted benzo[b]thiophene-5,6dicarboximides described in this paper. 


\section{Results and Discussion}

Commercially available thiophene-2,3-dicarbaldehyde (5) was subjected to the annulation reaction with various $N$-substituted maleimides in the presence of tri- $n$-octylphosphine in refluxing dioxane. Various $N$-substituted benzo[b]thiophene-5,6-dicarboximides (6a-i) were obtained in good to high yields. The results are shown in Table 1, indicating that both $N$-alkyl- and $N$-aryl-maleimides serve as a reactant for the annulation. Also, annulation of dialdehyde 5 with bismaleimides 7 and 8 provided bis(benzo[ $b]$ thiophene-5,6-dicarboximide)s 9 and 10 in good yields (Figure 3 ). It should be noted that most of all reactions completed for a short reaction time and the products were isolated directly from the reaction mixtures just by filtration. In literature there is only one reference, which described synthesis of benzo[b]thiophene-5,6-dicarboximides (Fig. 4). [13] Although this method is seems to be useful for preparing the 9-substituted compound, it requires an expensive samarium reagent and the yield of the two-pot procedures is moderate. Our method described in this paper is superior to the reported one in points of simplicity and efficiency of the reaction. Optical properties of some products are shown in
Table 2. The $N$-4-methoxyphenyl derivative $6 \mathrm{~g}$ shows clear dual emission in acetonitrile as seen in the spectra of $\mathrm{N}$-arylnaphthalene-2,3-dicarboximides. [14] Emission quantum yields of 6 are smaller than those of the corresponding naphthalene-2,3-dicarboxyimides 2, [15] probably because of a heavy atom effect of the sulfur atom. [16]

Table 1. Reaction time and yields of $N$-substituted benzo[b]thiophene5,6-dicarboximides $(6 a-j)$.

\begin{tabular}{lllll}
\hline entry & $\mathbf{R}$ & reaction time & product & yield (\%) \\
\hline 1 & $\mathrm{Me}$ & 1 & $6 \mathrm{a}$ & 86 \\
2 & $\mathrm{Et}$ & 1 & $6 \mathrm{~b}$ & 86 \\
3 & $c$-hexyl & 3 & $6 \mathrm{c}$ & 78 \\
4 & $\mathrm{Ph}$ & 1 & $6 \mathrm{~d}$ & 83 \\
5 & $4-\mathrm{Br}^{-} \mathrm{C}_{6} \mathrm{H}_{4}-$ & 3 & $6 \mathrm{e}$ & 93 \\
6 & $4-\mathrm{I}-\mathrm{C}_{6} \mathrm{H}_{4}-$ & 4 & $6 \mathrm{f}$ & 71 \\
7 & $4-\mathrm{MeO}^{-} \mathrm{C}_{6} \mathrm{H}_{4}-$ & 2 & $6 \mathrm{~g}$ & 90 \\
8 & $4-\mathrm{NO}_{2}-\mathrm{C}_{6} \mathrm{H}_{4}-$ & 2 & $6 \mathrm{~h}$ & 89 \\
9 & $4-\mathrm{Ph}_{-} \mathrm{C}_{6} \mathrm{H}_{4}-$ & 2 & $6 \mathrm{i}$ & 88 \\
10 & $3-\mathrm{pyridyl}^{-}$ & 2 & $6 \mathrm{j}$ & 75 \\
\hline
\end{tabular}<smiles>CN1C(=O)C=CC1=O</smiles><smiles>O=C1C=CC(=O)N1c1cccc(N2C(=O)C=CC2=O)c1</smiles>

8<smiles>O=Cc1ccsc1C=O</smiles>

5

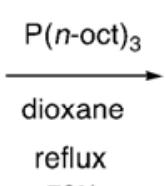

$73 \%$<smiles>O=C1c2cc3ccsc3cc2C(=O)N1c1cccc(N2C(=O)c3cc4ccsc4cc3C2=O)c1</smiles>

10

Figure 3. Synthesis of bis(benzo[b]thiophene-5,6-dicarboximide)s 9 and 10.<smiles>O=Cc1ccsc1</smiles>

11<smiles>Cc1ccc(C=O)cc1</smiles>

12
1. $\mathrm{Sml}_{2}, \mathrm{HMPA}$

2. $\mathrm{H}^{+}$, oxidation

$\sim 40 \%$<smiles>Cc1ccc(C(O)c2sccc2C=O)cc1</smiles>

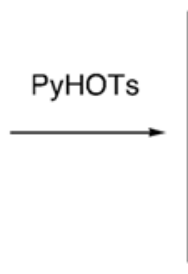<smiles>Cc1ccc(-c2occ3ccsc23)cc1</smiles>

14<smiles>Cc1ccc(C23OC(c4cccs4)C2C2C(=O)N(c4ccccc4)C(=O)C23)cc1</smiles>

15

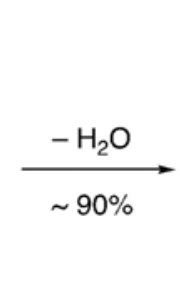<smiles>Cc1ccc(-c2c3c(cc4ccsc24)C(=O)N(c2ccccc2)C3=O)cc1</smiles>

16

Figure 4. A previously reported synthetic route to benzo[b]thiophene-5,6-dicarboximide derivatives. 
Table 2. Optical properties of selected benzo[b]thiophene-5,6-dicarboximides 6.

\begin{tabular}{|c|c|c|c|c|c|c|}
\hline Imides & solvent & $\lambda_{\text {abs }} / \mathrm{nm}$ & $\log \varepsilon$ & $\lambda_{\mathrm{emi}} / \mathbf{n m}$ & Stokes shift / nm & $\Phi / \%$ \\
\hline $6 a$ & $\mathrm{CH}_{3} \mathrm{OH}$ & 344 & 3.77 & 461 & 117 & 4 \\
\hline $6 b$ & $\mathrm{CHCl}_{3}$ & 348 & 3.79 & $\begin{array}{l}379 \\
413 \mathrm{sh}\end{array}$ & $\begin{array}{l}31 \\
65\end{array}$ & 3 \\
\hline $6 \mathrm{c}$ & $\mathrm{CHCl}_{3}$ & 348 & 3.78 & $\begin{array}{l}380 \\
409 \text { sh }\end{array}$ & $\begin{array}{l}32 \\
61\end{array}$ & 2 \\
\hline $6 \mathrm{~d}$ & $\mathrm{CH}_{3} \mathrm{CN}$ & 347 & 3.50 & 461 & 114 & 0.3 \\
\hline $\begin{array}{l}6 d \\
6 e\end{array}$ & $\begin{array}{l}\mathrm{CHCl}_{3} \\
\mathrm{CH}_{3} \mathrm{CN}\end{array}$ & $\begin{array}{l}350 \\
347\end{array}$ & $\begin{array}{l}3.83 \\
3.86\end{array}$ & $\begin{array}{l}477 \\
485\end{array}$ & $\begin{array}{l}127 \\
138\end{array}$ & $\begin{array}{l}0.6 \\
0.2\end{array}$ \\
\hline $6 \mathrm{f}$ & $\mathrm{CH}_{3} \mathrm{CN}$ & 347 & 3.81 & $\begin{array}{l}415 \\
513\end{array}$ & $\begin{array}{l}68 \\
166\end{array}$ & 0.3 \\
\hline $6 \mathrm{~g}$ & $\mathrm{CH}_{3} \mathrm{CN}$ & 348 & 3.80 & $\begin{array}{l}415 \\
513\end{array}$ & $\begin{array}{l}67 \\
165\end{array}$ & 0.3 \\
\hline $6 \mathrm{~g}$ & $\mathrm{CHCl}_{3}$ & 349 & 3.85 & 514 & 165 & 0.03 \\
\hline
\end{tabular}

Next, we turned our attention to derivatization of the product in order to increase the quantum yield and to gain an insight to possibility for tuning absorption and emission wavelengths. Introduction of cyano and aryl groups to the thiophene moiety of $6 \mathrm{c}$ was examined via its bromo derivative. Since Lemaire et al. reported an efficient access to 2-aryl derivatives from benzo[b]thiophene itself, we applied their methodology to chemical modification in our work. [17] At first, 3-bromo compound 17 was synthesized from 6c. Bromination of $6 \mathrm{c}$ with $\mathrm{N}$-bromosuccimide (NBS) in trifluoroacetic acid at $80^{\circ} \mathrm{C}$ for a short reaction time yielded 3-bromo derivative 7 in $96 \%$ yield, accompanied with a trace amount of 2,3-dibromo derivative 18. (Fig. 5) Although
Lemaire et al. found that benzo[b]thiophene can be brominated by NBS in refluxing acetic acid, the reaction of $6 \mathrm{c}$ under the same conditions in refluxing acetic acid required a longer reaction time and the yield of 17 was found to be moderate. It is stressed that using trifluoroacetic acid as a solvent in the reaction of $6 \mathrm{c}$ improved both the reaction time and the yield. Introduction of a cyano group was achieved by heating 17 with $\mathrm{CuCN}$ in dimethylsulfoxide (DMSO). [18] While reaction of 17 with an excess of $\mathrm{CuCN}$ in DMSO at $125^{\circ} \mathrm{C}$ for $12 \mathrm{~h}$ resulted in formation of 19 in $17 \%$ yield, accompanied with $53 \%$ of recovery, the reaction of 17 at $160^{\circ} \mathrm{C}$ for a shorter reaction time of $6 \mathrm{~h}$ provided 19 in $78 \%$ yield.

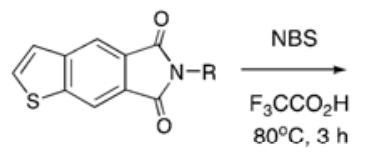<smiles>[R]N1C(=O)c2cc3scc(Br)c3cc2C1=O</smiles>

$17(96 \%)$

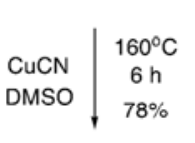

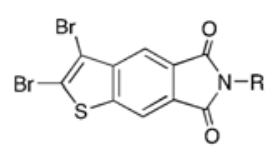

18 (trace)

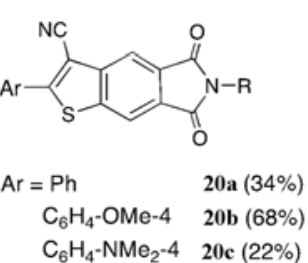

Figure 5. Derivatization of $N$-cyclohexyl derivative $6 c$.

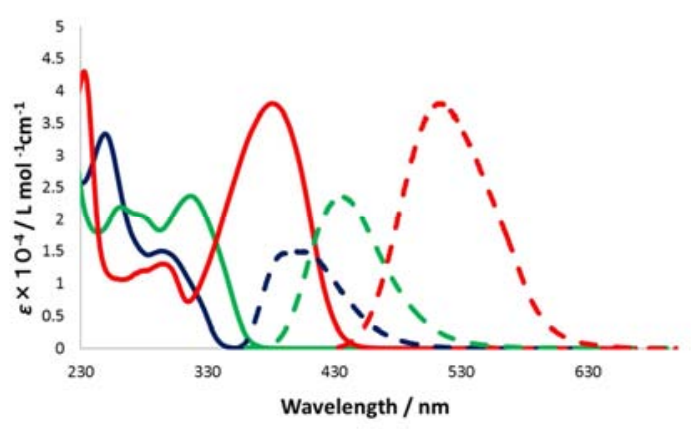

Figure 6. $U V$-vis absorption (solid line) and emission (broken line) spectra of 2-aryl-3-cyano-N-cyclohexylbenzo[b] thiophene-5,6-dicarboximides $20 \mathrm{a}$ (blue), $20 b$ (green) and 20c (red) in chloroform.
Finally, the Mizoroki-Heck reactions of 19 with various aryliodides in the presence of $\mathrm{Pd}(\mathrm{OAc})_{2}$, potassium carbonate and tetra- $n$-butylammonium bromide produced 2-aryl derivatives $20 \mathrm{a}-\mathrm{c}$. Optical properties of 19 and $20 \mathrm{a}-\mathrm{c}$ are summarized in Table 3. The UV-vis and emission spectra of $20 \mathrm{a}-\mathrm{c}$ are shown in Fig. 6.

Although 19 shows weaker emission quantum yield (1\%) than $6 \mathrm{c}$ does, 2-aryl derivatives $20 \mathrm{a}-\mathrm{c}$ indicate the enhanced quantum yields up to $27 \%$ in spite of existence of a heavy atom of sulfur. The absorptions shift with a hyperchromic effect depending on the substituents at the para position of the phenyl group and similarly emission wavelength shifts, clearly indicating that it is possible to tune both absorption and emission wavelengths of the title compounds by 
introduction of aryl groups with an electron-donating substituent at the 2 position.

Table 3. Optical properties of some N-cyclohexylbenzo[b]thiophene-5,6dicarboximides 19 and $20 a-c$ in chloroform.

\begin{tabular}{llllll}
\hline Imides & $\lambda_{\text {abs }} / \mathbf{n m}$ & $\log \varepsilon$ & $\lambda_{\text {emi }} / \mathbf{n m}$ & Stokes shift $/ \mathbf{n m}$ & $\boldsymbol{\Phi} / \mathbf{\%}$ \\
\hline 19 & 338 & 3.67 & 368 & 30 & 1 \\
$20 \mathrm{a}$ & $327 \mathrm{sh}$ & 4.18 & 405 & 78 & 6 \\
$20 \mathrm{~b}$ & $350 \mathrm{sh}$ & 4.37 & 436 & 86 & 17 \\
$20 \mathrm{c}$ & 414 & 4.58 & 511 & 97 & 27 \\
\hline
\end{tabular}

\section{Experimental}

\subsection{General Remarks}

Melting points were measured on a Yanaco MP-3 and are uncorrected. IR spectra were recorded on a JASCO FT/IR-4100 spectrometer and relative intensity is indicated with letters, vs, v, m, and w. as very strong, strong, medium, and weak, respectively. UV-vis spectra were recorded on a Shimadzu UV-2550 spectrometer. Emission spectra were recorded on a Shimadzu RF5300-PC spectrometer. Emission quantum yields were obtained by comparison with that of anthracene ( $\Phi=27 \%$ in ethanol). ${ }^{1} \mathrm{H}$ - and ${ }^{13} \mathrm{C}$-NMR spectra were recorded on JEOL $\lambda 400$ and ECA500 spectrometers. A chemical shift value of tetramethylsilane ( $\delta=0 \mathrm{ppm})$ for both ${ }^{1} \mathrm{H}$-NMR ${ }^{13} \mathrm{C}$-NMR spectra was used as internal standard. Mass spectra were measured on a JMS-700 mass spectrometer. Column chromatography was performed with Silica gel $60 \mathrm{~N}$ from Kanto Chem. DMSO, $N, N$-dimethylformamide (DMF), and 1,4-dioxane were purchased from Kanto Chem. and were distilled over $\mathrm{CaH}_{2}$. Dichloromethane, chloroform, trifluoroacetic acid, and acetonitrile were also purchased from Kanto Chem. Tri- $n$-octylphosphine, $N$-methylmaleimide, $N$ ethylmaleimide, $N$-cyclohexylmaleimide, $N$-phenylmaleimide, $N, N$ '-( $m$-phenylene)bismaleimide, $N$-bromosuccimide (NBS), $\mathrm{N}$-iodosuccimide (NIS), 4-iodoanisole, iodobenzene, and thiophene-2,3-dicarbaldehyde were purchased from Tokyo Chemical Industry, Inc. Copper(I) cyanide, palladium acetate, and tetra- $n$-butylammonium bromide were purchased from Wako Chem. $N$-(4-Methoxyphenyl)-, $N$-(4-bromophenyl)-, $N$-(4-iodophenyl)-, $\quad N$-( $p$-biphenylyl)-, and $N$-3-pyridylmaleimides were prepared according to a two-step procedure from maleic anhydride and corresponding amines reported by Cava et al. [19] $N, N^{\prime}$-Hexamethylenedimaleimide was prepared by the method of Tona et al. [20] 4-Iodo- $N, N$-dimethylaniline was prepared by NIS iodination of $N, N$-dimethylaniline. [21]

\subsection{General Procedure for Synthesis of Benzo[b]Thiophene-5,6-Dicarboximides 6}

To a solution of the thiophene-2,3-dicarbaldehyde (1.0 mmol) and $N$-substituted maleimide $(1.1 \mathrm{mmol})$ in $2 \mathrm{ml}$ of dry dioxane was added tri- $n$-octylphosphine $(1.2 \mathrm{mmol})$. The mixture was refluxed on a preheated oil bath under nitrogen atmosphere for $1-4 \mathrm{~h}$, and was cooled to room temperature. To the reaction mixture was added $2 \mathrm{ml}$ of hexane and the annulation product crystalized at ice-bath temperature. The crystals were collected by suction filtration and washed well with cold ether/hexane (1/1) to give a pure product.

6a: Colorless prisms, m.p. $224-226{ }^{\circ} \mathrm{C} .{ }^{1} \mathrm{HNMR}\left(\mathrm{CDCl}_{3}\right.$, $400 \mathrm{MHz}) \delta=8.33(\mathrm{~d}, J=0.8 \mathrm{~Hz}, 1 \mathrm{H}), 8.26(\mathrm{~s}, 1 \mathrm{H}), 7.76(\mathrm{dd}$, $J=6.4,0.8 \mathrm{~Hz}, 1 \mathrm{H}) 7.55(\mathrm{~d}, J=8.0 \mathrm{~Hz}, 1 \mathrm{H}), 3.22(\mathrm{~s}, 3 \mathrm{H}) \mathrm{ppm}$;

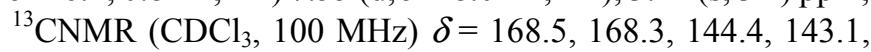
131.5, 128.4, 127.5, 125.0, 119.0, 118.4, 24.1ppm; IR (KBr) $v$ $=1764(\mathrm{~m}), 1692(\mathrm{vs}) \mathrm{cm}^{-1} ; \mathrm{UV}$-vis $\left(\mathrm{CH}_{3} \mathrm{OH}\right) \lambda_{\max }=215(\log \varepsilon$ $=4.11), 235$ (4.35), 256 (4.62), 328 (3.64), 344 (3.77) nm; MS (70 eV) $\mathrm{m} / \mathrm{z}(\%) 218(30), 217\left(\mathrm{M}^{+}, 100\right), 216(15), 189(33)$, 188 (17), 173 (76), 161 (19), 160 (28), 133 (25), 132 (60), 94 (19), 66 (11). HRMS Calcd for $\mathrm{C}_{11} \mathrm{H}_{7} \mathrm{NO}_{2} \mathrm{~S}\left(\mathrm{M}^{+}\right)$217.0198, found 217.0198 .

6b: Colorless plates, m.p. $171-172{ }^{\circ} \mathrm{C} .{ }^{1} \mathrm{HNMR}\left(\mathrm{CDCl}_{3}\right.$, $400 \mathrm{MHz}) \delta=8.33(\mathrm{t}, J=0.7 \mathrm{~Hz}, 1 \mathrm{H}), 8.26(\mathrm{~d}, J=0.7 \mathrm{~Hz}, 1 \mathrm{H})$, $7.75(\mathrm{~d}, J=5.5 \mathrm{~Hz}, 1 \mathrm{H}), 7.55(\mathrm{dd}, J=5.5,0.7 \mathrm{~Hz}, 1 \mathrm{H}), 3.79(\mathrm{q}$, $J=7.3 \mathrm{~Hz}, 2 \mathrm{H}), 1.31(\mathrm{t}, J=7.3 \mathrm{~Hz}, 3 \mathrm{H}) \mathrm{ppm} ;{ }^{13} \mathrm{CNMR}$ $\left(\mathrm{CDCl}_{3}, 100 \mathrm{MHz}\right) \delta=168.2,168.1,144.4,143.1,131.4$, 128.4, 127.6, 125.0, 119.0, 118.4, 33.1, 14.0 ppm; IR (KBr) $v$ $=1761(\mathrm{~s}), 1746(\mathrm{~s}), 1695(\mathrm{vs}) \mathrm{cm}^{-1} ; \mathrm{MS}(70 \mathrm{eV}) \mathrm{m} / \mathrm{z}(\%) 231$ $\left(\mathrm{M}^{+}, 56\right), 216$ (100), 203 (8), 189 (9), 161 (12), 132 (17). HRMS Calcd for $\mathrm{C}_{12} \mathrm{H}_{9} \mathrm{NO}_{2} \mathrm{~S}\left(\mathrm{M}^{+}\right)$231.0354, found 231.0354.

6c: Colorless microcrystals, m.p. $232-233^{\circ} \mathrm{C} .{ }^{1} \mathrm{HNMR}$ $\left(\mathrm{CDCl}_{3}, 500 \mathrm{MHz}\right) \delta=8.30(\mathrm{t}, J=0.9 \mathrm{~Hz}, 1 \mathrm{H}), 8.23(\mathrm{~d}, J=0.9$ $\mathrm{Hz}, 1 \mathrm{H}), 7.73(\mathrm{~d}, J=5.5 \mathrm{~Hz}, 1 \mathrm{H}), 7.54(\mathrm{dd}, J=5.5,0.9 \mathrm{~Hz}$, $1 \mathrm{H}), 4.15(\mathrm{tt}, J=12.8,3.7 \mathrm{~Hz}, 1 \mathrm{H}), 2.25(\mathrm{qd}, J=12.8,3.7 \mathrm{~Hz}$, $2 \mathrm{H}), 1.88(\mathrm{dm}, J=12.8 \mathrm{~Hz}, 2 \mathrm{H}), 1.76(\mathrm{dm}, J=12.8 \mathrm{~Hz}, 2 \mathrm{H})$, $1.70(\mathrm{dm}, J=12.8 \mathrm{~Hz}, 1 \mathrm{H}), 1.39(\mathrm{qt}, J=12.8,3.7 \mathrm{~Hz}, 2 \mathrm{H})$, 1.29 (qt, $J=12.8,3.7 \mathrm{~Hz}, 1 \mathrm{H}) \mathrm{ppm} ;{ }^{13} \mathrm{CNMR}\left(\mathrm{CDCl}_{3}, 126\right.$ MHz) $\delta=168.4168 .3,144.3,143.1,131.2,128.3,127.5$, 125.0, 118.8, 118.2, 51.1, 29.9, 26.1, $25.1 \mathrm{ppm}$; IR (KBr) $v=$ $1715(\mathrm{~s}), 1700(\mathrm{vs}), 1684(\mathrm{~s}) \mathrm{cm}^{-1} ; \mathrm{UV}$-vis $\left(\mathrm{CHCl}_{3}\right) \lambda_{\max }=260$ $(\log \varepsilon=4.70), 333(3.70), 348(3.78) \mathrm{nm} ; \mathrm{MS}(70 \mathrm{eV}) \mathrm{m} / \mathrm{z}(\%)$ $285\left(\mathrm{M}^{+}, 61\right), 242$ (38), 204 (100), $186(25), 161$ (10), 132 (14). HRMS Calcd for $\mathrm{C}_{16} \mathrm{H}_{15} \mathrm{NO}_{2} \mathrm{~S}\left(\mathrm{M}^{+}\right)$285.08235, found 285.0828.

6d: Creamy white microcrystals, m.p. $250-251^{\circ} \mathrm{C} .{ }^{1} \mathrm{HNMR}$ $\left(\mathrm{CDCl}_{3}, 500 \mathrm{MHz}\right) \delta=8.46(\mathrm{t}, J=0.9 \mathrm{~Hz}, 1 \mathrm{H}), 8.38(\mathrm{~d}, J=0.9$ $\mathrm{Hz}, 1 \mathrm{H}), 7.80(\mathrm{~d}, J=5.5 \mathrm{~Hz}, 1 \mathrm{H}), 7.60(\mathrm{dd}, J=5.5,0.6 \mathrm{~Hz}, 1 \mathrm{H})$ $7.50(\mathrm{~m}, 4 \mathrm{H}), 7.42(\mathrm{tm}, J=6.5 \mathrm{~Hz}, 1 \mathrm{H}) \mathrm{ppm} ;{ }^{13} \mathrm{CNMR}\left(\mathrm{CDCl}_{3}\right.$, $126 \mathrm{MHz}) \delta=167.4,167.3,145.0,143.6,132.02,131.98$, 129.2, 128.2, 128.0, 127.1, 126.7, 125.2, 119.7, 119.2 ppm; IR (KBr) $v=1774(\mathrm{~s}), 1707$ (vs) cm $\mathrm{cm}^{-1}$, UV-vis $\left(\mathrm{CH}_{3} \mathrm{OH}\right) \lambda_{\max }=$ $234(\log \varepsilon=4.26), 260(4.61), 345(3.66) \mathrm{nm} ; \mathrm{MS}(70 \mathrm{eV}) \mathrm{m} / \mathrm{z}$ (\%) 280 (18), $279\left(\mathrm{M}^{+}, 100\right), 236$ (10), 235 (57), 132 (25). HRMS Calcd for $\mathrm{C}_{16} \mathrm{H}_{9} \mathrm{NO}_{2} \mathrm{~S}\left(\mathrm{M}^{+}\right) 279.0354$, found 279.0354 .

6e: Colorless microcrystals, m.p. $>300{ }^{\circ} \mathrm{C} .{ }^{1} \mathrm{HNMR}$ (DMSO- $d_{6}$ at $\left.130^{\circ} \mathrm{C}, 500 \mathrm{MHz}\right) \delta=8.62(\mathrm{~s}, 1 \mathrm{H}), 8.44(\mathrm{~s}, 1 \mathrm{H})$, $8.12(\mathrm{~d}, J=5.5 \mathrm{~Hz}, 1 \mathrm{H}), 7.75(\mathrm{~d}, J=5.5 \mathrm{~Hz}, 1 \mathrm{H}), 7.70(\mathrm{~d}, J=$ $8.0 \mathrm{~Hz}, 1 \mathrm{H}), 7.46(\mathrm{~d}, J=8.0 \mathrm{~Hz}, 1 \mathrm{H}) \mathrm{ppm} ;{ }^{13} \mathrm{CNMR}$ (DMSO- $d_{6}$ at $\left.130^{\circ} \mathrm{C}, 126 \mathrm{MHz}\right) \delta=165.9,165.8,144.1,143.1$, 132.7, 131.2, 131.2, 128.4, 127.1, 126.1, 124.6, 120.2, 118.8, $118.6 \mathrm{ppm}$; IR (KBr) $v=1784(\mathrm{~m}), 1717$ (vs) $\mathrm{cm}^{-1}$; UV-vis $\left(\mathrm{CH}_{3} \mathrm{CN}\right) \lambda_{\max }=228(\log \varepsilon=4.43), 262(4.81), 334$ sh (3.78), 347 (3.86) nm; MS (70 eV) m/z (\%) $359\left(\mathrm{M}^{+}, 100\right), 357\left(\mathrm{M}^{+}\right.$, 97), 315 (33), 160 (10), 139 (15), 132 (15). HRMS Calcd for 
$\mathrm{C}_{16} \mathrm{H}_{8}{ }^{79} \mathrm{BrNO}_{2} \mathrm{~S}\left(\mathrm{M}^{+}\right), 356.9459$, found 356.9458 .

6f: Creamy white microcrystals, m.p. $>300{ }^{\circ} \mathrm{C}$. ${ }^{1} \mathrm{HNMR}$ (DMSO- $d_{6}$ at $\left.130^{\circ} \mathrm{C}, 400 \mathrm{MHz}\right) \delta=8.28(\mathrm{~s}, 1 \mathrm{H}), 8.04(\mathrm{~s}, 1 \mathrm{H})$, $7.74(\mathrm{~d}, J=5.6 \mathrm{~Hz}, 1 \mathrm{H}), 7.46(\mathrm{dm}, J=8.4 \mathrm{~Hz}, 2 \mathrm{H}), 7.34(\mathrm{~d}, J=$ $5.2 \mathrm{~Hz}, 1 \mathrm{H}), 6.86(\mathrm{dm}, J=8.4 \mathrm{~Hz}, 2 \mathrm{H}) \mathrm{ppm} ;{ }^{13} \mathrm{CNMR}$ $\left(\mathrm{DMSO}-d_{6}\right.$ at $\left.130^{\circ} \mathrm{C}, 126 \mathrm{MHz}\right) \delta=165.8,165.7,144.1,143.0$ 137.1, 132.5, 131.7, 128.4, 127.1, 126.1, 124.6, 118.7, 118.5, 116.1 ppm; IR (KBr) $v=1718$ (vs), 1709 (vs), 1679 (s) $\mathrm{cm}^{-1}$; UV-vis $\left(\mathrm{CH}_{3} \mathrm{CN}\right) \lambda_{\max }=231(\log \varepsilon=4.39), 263$ (4.76), 334 (3.74), 347 (3.81) nm; MS (70 eV) m/z (\%) 406 (19), $405\left(\mathrm{M}^{+}\right.$, 100), 361 (23), 234 (23), 132 (45). HRMS Calcd for $\mathrm{C}_{16} \mathrm{H}_{15} \mathrm{NIO}_{2} \mathrm{~S}\left(\mathrm{M}^{+}\right)$404.9320, found 404.9321.

6g: Yellowish microcrystals, m.p. $228-229{ }^{\circ} \mathrm{C} .{ }^{1} \mathrm{HNMR}$ $\left(\mathrm{CDCl}_{3}, 500 \mathrm{MHz}\right) \delta=8.45(\mathrm{~s}, 1 \mathrm{H}), 8.36(\mathrm{~s}, 1 \mathrm{H}), 7.79(\mathrm{~d}, J=$ $5.2 \mathrm{~Hz}, 1 \mathrm{H}), 7.59(\mathrm{~d}, J=5.2 \mathrm{~Hz}, 1 \mathrm{H}), 7.38(\mathrm{~d}, J=9.0 \mathrm{~Hz}, 2 \mathrm{H})$, $7.03(\mathrm{~d}, J=9.0 \mathrm{~Hz}, 2 \mathrm{H}), 3.86(\mathrm{~s}, 3 \mathrm{H}) \mathrm{ppm} ;{ }^{13} \mathrm{CNMR}\left(\mathrm{CDCl}_{3}\right.$, $126 \mathrm{MHz}) \delta=167.5,167.4,159.2,144.7,143.4,131.7,127.92$, $127.89,127.1,125.0,124.5,119.5,118.9,114.4,55.5$ ppm; IR $(\mathrm{KBr}) \boldsymbol{v}=1769(\mathrm{~s}), 1714$ (vs) $\mathrm{cm}^{-1}$; UV-vis $\left(\mathrm{CH}_{3} \mathrm{CN}\right) \lambda_{\max }=$ $228(\log \varepsilon=4.46), 260$ (4.66), 334 (3.74), 345 (3.80) nm; MS (70 eV) $m / z(\%) 310(20), 309\left(\mathrm{M}^{+}, 100\right), 294$ (40), 265 (9), 186 (9), 132 (11). HRMS Calcd for $\mathrm{C}_{17} \mathrm{H}_{11} \mathrm{NO}_{3} \mathrm{~S}\left(\mathrm{M}^{+}\right)$ 309.0460, found 309.0461.

6h: Colorless microcrystals, m.p. $>300 \quad{ }^{\circ} \mathrm{C} .{ }^{1} \mathrm{HNMR}$ (DMSO- $d_{6}$ at $\left.130^{\circ} \mathrm{C}, 500 \mathrm{MHz}\right) \delta=8.67(\mathrm{~s}, 1 \mathrm{H}), 8.49(\mathrm{~s}, 1 \mathrm{H})$, $8.36(\mathrm{~d}, J=8.4 \mathrm{~Hz}, 2 \mathrm{H}), 8.15(\mathrm{~d}, J=5.2 \mathrm{~Hz}, 1 \mathrm{H}), 7.86(\mathrm{~d}, J=$ $8.4 \mathrm{~Hz}, 2 \mathrm{H}), 7.78(\mathrm{~d}, J=5.2 \mathrm{~Hz}, 1 \mathrm{H}) \mathrm{ppm} ;{ }^{13} \mathrm{CNMR}$ (DMSO- $d_{6}$ at $\left.130^{\circ} \mathrm{C}, 126 \mathrm{MHz}\right) \delta=165.5,165.4,146.0,144.3$, 143.2, 137.5, 132.8, 126.9, 126.7, 125.9, 124.6, 123.3, 118.9, 118.7 ppm; IR (KBr) $v=1765$ (s), 1729 (vs) cm $\mathrm{cm}^{-1}$; MS $(70 \mathrm{eV})$ $\mathrm{m} / \mathrm{z}$ (\%) $324\left(\mathrm{M}^{+}, 100\right), 294$ (25), 280 (24), 234 (22), 222 (10), 160 (14), 132 (45). HRMS Calcd for $\mathrm{C}_{16} \mathrm{H}_{8} \mathrm{~N}_{2} \mathrm{O}_{4} \mathrm{~S}\left(\mathrm{M}^{+}\right)$ 324.0205 , found 324.0207 .

6i: Yellowish leaflets, m.p. $>300{ }^{\circ} \mathrm{C}$. ${ }^{1} \mathrm{HNMR}$ (DMSO- $d_{6}$ at $\left.130^{\circ} \mathrm{C}, 500 \mathrm{MHz}\right) \delta=8.63(\mathrm{~s}, 1 \mathrm{H}), 8.46(\mathrm{~s}, 1 \mathrm{H}), 8.12(\mathrm{~d}, J=$ $5.6 \mathrm{~Hz}, 1 \mathrm{H}), 7.78(\mathrm{~m}, 3 \mathrm{H}), 7.71(\mathrm{~d}, J=8.4 \mathrm{~Hz}, 2 \mathrm{H}), 7.58(\mathrm{~d}, J$ $=8.4 \mathrm{~Hz}, 2 \mathrm{H}), 7.49(\mathrm{t}, J=7.3 \mathrm{~Hz}, 2 \mathrm{H}), 7.39(\mathrm{t}, J=7.3 \mathrm{~Hz}, 1 \mathrm{H})$ ppm; ${ }^{13} \mathrm{CNMR}$ (DMSO- $d_{6}$ at $\left.130{ }^{\circ} \mathrm{C}, 126 \mathrm{MHz}\right) \delta=166.2$, $166.1,144.1,143.1,139.5,139.1,132.5,131.1,128.3,127.2$, 127.0, 126.7, 126.5, 126.3, 126.2, 124.6, 118.7, 118.4 ppm; IR $(\mathrm{KBr}) v=1774(\mathrm{~m}), 1703(\mathrm{vs}) \mathrm{cm}^{-1}$; MS $(70 \mathrm{eV}) \mathrm{m} / z(\%) 356$ (25), $355\left(\mathrm{M}^{+}, 100\right), 311$ (20), 152 (13), 132 (22). HRMS Calcd for $\mathrm{C}_{22} \mathrm{H}_{13} \mathrm{NO}_{2} \mathrm{~S}\left(\mathrm{M}^{+}\right)$355.0667, found 355.0670.

6j: Yellowish microcrystals, m.p. $>300{ }^{\circ} \mathrm{C} .{ }^{1} \mathrm{HNMR}$ $\left(\mathrm{DMSO}-d_{6}\right.$ at $\left.130^{\circ} \mathrm{C}, 400 \mathrm{MHz}\right) \delta=8.76(\mathrm{~d}, J=0.7 \mathrm{~Hz}, 1 \mathrm{H})$, $8.72(\mathrm{~d}, J=2.0 \mathrm{~Hz}, 1 \mathrm{H}), 8.63(\mathrm{dm}, J=4.8 \mathrm{~Hz}, 1 \mathrm{H}), 8.52 \mathrm{~d}, J=$ $0.7 \mathrm{~Hz}, 1 \mathrm{H}), 8.20(\mathrm{~d}, J=5.5 \mathrm{~Hz}, 1 \mathrm{H}), 7.96(\mathrm{ddm}, J=8.1,2.0$ $\mathrm{Hz}, 1 \mathrm{H}), 7.80$ (d, $J=5.5 \mathrm{~Hz}, 1 \mathrm{H}), 7.60$ (dd, $J=8.1,4.8 \mathrm{~Hz}, 1 \mathrm{H})$ ppm; ${ }^{13} \mathrm{CNMR}$ (DMSO- $d_{6}$ at $\left.130^{\circ} \mathrm{C}, 100 \mathrm{MHz}\right) \delta=165.9$, 165.8, 148.0, 147.0, 144.1, 143.1, 133.7, 132.7, 128.7, 127.1, $126.1,124.6,123.0,118.8,118.6 \mathrm{ppm} ; \mathrm{IR}(\mathrm{KBr}) \boldsymbol{v}=1783(\mathrm{~m})$, $1708(\mathrm{vs}) \mathrm{cm}^{-1}$; UV-vis $\left(\mathrm{CH}_{3} \mathrm{CN}\right) \lambda_{\max }=230(\log \varepsilon=4.28), 261$ (4.71), 334 (3.70), 347 (3.79) nm; MS (70 eV) m/z (\%) 281 (18), $280\left(\mathrm{M}^{+}, 100\right), 236$ (53), 235 (44), 210 (11), 159 (11), 132 (61). HRMS Calcd for $\mathrm{C}_{15} \mathrm{H}_{8} \mathrm{~N}_{2} \mathrm{O}_{2} \mathrm{~S}\left(\mathrm{M}^{+}\right) 280.0307$, found 280.0309 .

\subsection{General Procedure for Synthesis of Bis(Benzo[b]- Thiophene-5,6-Dicarboximide)s 9 and 10}

To a solution of thiophene-2,3-dicarbaldehyde (1.0 mmol) and $N$-substituted maleimide $(0.55 \mathrm{mmol})$ in $2 \mathrm{ml}$ of dry dioxane was added tri- $n$-octylphosphine $(1.2 \mathrm{mmol})$. The mixture was refluxed on a preheated oil bath under nitrogen atmosphere for $4 \mathrm{~h}$, and was cooled to ice-bath temperature. The crystals formed were collected by suction filtration and washed well with cold ether/hexane (1/1) to give a product.

9: Brownish solids, m.p. $257-258{ }^{\circ} \mathrm{C} .{ }^{1} \mathrm{HNMR}\left(\mathrm{CDCl}_{3}, 500\right.$ $\mathrm{MHz}) \delta=8.31(\mathrm{t}, J=0.8 \mathrm{~Hz}, 2 \mathrm{H}), 8.24(\mathrm{~d}, J=0.8 \mathrm{~Hz}, 2 \mathrm{H})$, $7.74(\mathrm{~d}, J=5.5 \mathrm{~Hz}, 2 \mathrm{H}), 7.54$ (dd, $J=5.5,0.8 \mathrm{~Hz}, 2 \mathrm{H}), 3.71(\mathrm{t}$, $J=7.3 \mathrm{~Hz}, 4 \mathrm{H}$ ), 1.71 (quin, $J=7.3 \mathrm{~Hz}, 4 \mathrm{H}$ ), 1.41 (quin, $J=7.3$

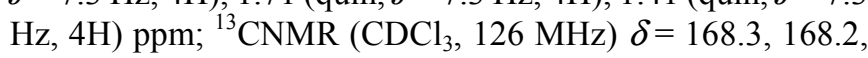
$144.3,143.0,131.3,128.3,127.5,125.0,119.0,118.4,38.0$, 28.5, 26.5 ppm; IR (KBr) $v=1758$ (s), 1699 (vs) $\mathrm{cm}^{-1}$; MS $m / z(\%)=488\left(\mathrm{M}^{+}, 48\right), 272$ (22), 230 (10), 216 (100), 204 (10), 186 (10), 161 (10). HRMS Calcd for $\mathrm{C}_{26} \mathrm{H}_{20} \mathrm{~N}_{2} \mathrm{O}_{4} \mathrm{~S}_{2}\left(\mathrm{M}^{+}\right)$ 488.0865, found 488.0860.

10: Brownish solids, m.p. $>300{ }^{\circ} \mathrm{C}$. ${ }^{1} \mathrm{HNMR}$ (DMSO- $d_{6}$ at $\left.100^{\circ} \mathrm{C}, 500 \mathrm{MHz}\right) \delta=8.67(\mathrm{~s}, 2 \mathrm{H}), 8.47(\mathrm{~s}, 2 \mathrm{H}), 8.14(\mathrm{~d}, J=$ $5.6 \mathrm{~Hz}, 2 \mathrm{H}), 7.77$ (d, $J=5.6 \mathrm{~Hz}, 2 \mathrm{H}), 7.68(\mathrm{~m}, 2 \mathrm{H}), 7.58(\mathrm{~m}$, $2 \mathrm{H}) \mathrm{ppm} ;{ }^{13} \mathrm{CNMR}$ (DMSO- $d_{6}$ at $\left.100^{\circ} \mathrm{C}, 126 \mathrm{MHz}\right) \delta=$ $165.94,165.85,144.1,143.1,132.5,132.3,128.4,127.1$, $126.2,125.8,124.8,124.6,118.7,118.5 \mathrm{ppm}$; IR (KBr) $v=$ 1770 (s), 1722 (vs) cm $\mathrm{cm}^{-1}$ MS $m / z(\%)=481(31), 480\left(\mathrm{M}^{+}\right.$, 100), 240 (8), 132 (22). HRMS Calcd for $\mathrm{C}_{26} \mathrm{H}_{12} \mathrm{~N}_{2} \mathrm{O}_{4} \mathrm{~S}_{2}\left(\mathrm{M}^{+}\right)$ 480.0239 , found 480.0242 .

\subsection{3-Bromo-N-CyclohexylBenzo[b]Thiophene-5,6-Di- carboximide (17)}

A mixture of $285 \mathrm{mg}$ (1.00 mmol) of $6 \mathrm{c}$ and $890 \mathrm{mg}(5.00$ $\mathrm{mmol}$ ) of NBS in $15 \mathrm{~mL}$ of trifluoroacetic acid was heated on an oil bath for $3 \mathrm{~h}$. The reaction mixture was poured into water and was extracted with ether $(30 \mathrm{ml} \times 3)$. The combined organic layer was washed with a saturated $\mathrm{NaHCO}_{3}$ aqueous solution and brine. After dryness over $\mathrm{Na}_{2} \mathrm{SO}_{4}$, the solvent was evaporated and the residue was purified by silica gel chromatography $\left(\mathrm{CHCl}_{3} /\right.$ hexane $\left.=3 / 1\right)$ to give $252 \mathrm{mg}$ of 17 $(96 \%)$ as colorless microcrystals, followed by a trace amount of 18. Independently, 18 was obtained as colorless microcrystals by NBS bromination for a longer reaction time (14 h) from 17 in $84 \%$ yield.

17: M.p. $221-223^{\circ} \mathrm{C} .{ }^{1} \mathrm{HNMR}\left(\mathrm{CDCl}_{3}, 500 \mathrm{MHz}\right) \delta=8.28$ $(\mathrm{d}, J=0.7 \mathrm{~Hz}, 1 \mathrm{H}), 8.27$ (d, $J=0.7 \mathrm{~Hz} .1 \mathrm{H}), 7.71(\mathrm{~s}, 1 \mathrm{H}), 4.17$ (tt, $J=12.4,3.7 \mathrm{~Hz}, 1 \mathrm{H}), 2.25(\mathrm{qd}, J=12.4,3.7 \mathrm{~Hz}, 2 \mathrm{H}), 1.89$ $(\mathrm{dm}, J=12.4 \mathrm{~Hz}, 2 \mathrm{H}), 1.77(\mathrm{dm}, J=12.4 \mathrm{~Hz}, 2 \mathrm{H}), 1.71(\mathrm{dm}, J$ $=12.4 \mathrm{~Hz}, 1 \mathrm{H}), 1.25-1.44(\mathrm{~m}, 3 \mathrm{H}) \mathrm{ppm} ;{ }^{13} \mathrm{CNMR}\left(\mathrm{CDCl}_{3}\right.$, $126 \mathrm{MHz}) \delta=168.03,167.97,143.6,141.5,129.1,128.6$, $128.3,118.8,118.5,109.4,51.4,30.0,26.2,25.3$ ppm; IR (KBr) $v=1766(\mathrm{~m}), 1702(\mathrm{vs}) \mathrm{cm}^{-1}$; MS (70 eV) $\mathrm{m} / z(\%) 365$ $\left(\mathrm{M}^{+}, 57\right), 363\left(\mathrm{M}^{+}, 55\right), 322(32), 320$ (30), 285 (14), $284(100)$, 283 (27), 282 (98), 281 (15), 266 (23), 264 (22), 239 (12), 212 (13), 210 (13). HRMS Calcd for $\mathrm{C}_{16} \mathrm{H}_{14}{ }^{79} \mathrm{BrNO}_{2} \mathrm{~S}\left(\mathrm{M}^{+}\right)$, 362.9927 , found 362.9926 .

18: M.p. $277-279{ }^{\circ} \mathrm{C} .{ }^{1} \mathrm{HNMR}\left(\mathrm{CDCl}_{3}, 400 \mathrm{MHz}\right) \delta=8.17$ 
$(\mathrm{d}, J=0.4 \mathrm{~Hz}, 1 \mathrm{H}), 8.13(\mathrm{~d}, J=0.4 \mathrm{~Hz}, 1 \mathrm{H}), 4.13(\mathrm{tt}, J=12.6$, $3.4 \mathrm{~Hz}, 1 \mathrm{H}), 2.21(\mathrm{qd}, J=12.6,3.4 \mathrm{~Hz}, 2 \mathrm{H}), 1.86(\mathrm{dm}, J=12.6$ $\mathrm{Hz}, 2 \mathrm{H}), 1.73(\mathrm{dm}, J=12.6 \mathrm{~Hz}, 2 \mathrm{H}), 1.69(\mathrm{dm}, J=12.6 \mathrm{~Hz}$, 1H), $1.41-1.21(\mathrm{~m}, 3 \mathrm{H}) \mathrm{ppm} ;{ }^{13} \mathrm{CNMR}\left(\mathrm{CDCl}_{3}, 100 \mathrm{MHz}\right) \delta=$ 167.6, 143.6, 141.3, 129.3, 128.6, 119.0, 118.6, 117.4, 113.3, 54.3, 51.3, 29.8, 26.0, 25.1 ppm; IR (KBr) $v=1765$ (s), 1705 (vs) $\mathrm{cm}^{-1}$; MS (70 eV) m/z (\%) $445\left(\mathrm{M}^{+}, 31\right), 443\left(\mathrm{M}^{+}, 59\right)$, $441\left(\mathrm{M}^{+}, 29\right), 402$ (16), 400 (29), 398 (15), 364 (53), 363 (26), 362 (100), 344 (20), 342 (20), 319 (10), 290 (13). HRMS Calcd for $\mathrm{C}_{16} \mathrm{H}_{13}{ }^{79} \mathrm{Br}_{2} \mathrm{NO}_{2} \mathrm{~S}\left(\mathrm{M}^{+}\right), 440.9034$, found 440.9036 .

\subsection{3-Cyano-N-CyclohexylBenzo[b]Thiophene-5, 6-Dicarboximide (19)}

A mixture of $182 \mathrm{mg}(0.500 \mathrm{mmol})$ of 17 and $134 \mathrm{mg}(1.50$ mmol, 3.0 eq.) of $\mathrm{CuCN}$ in $5 \mathrm{~mL}$ of dry DMSO was heated on an oil bath at $125^{\circ} \mathrm{C}$ for $12 \mathrm{~h}$. The reaction mixture was cooled to room temperature and was passed through a Celite ${ }^{\circledR}$ pad. The filtrate was poured into water and was extracted with ether $(20 \mathrm{ml} \times 3)$. The combined organic layer was washed with a saturated $\mathrm{NaHCO}_{3}$ aqueous solution and brine. After dryness over $\mathrm{Na}_{2} \mathrm{SO}_{4}$, the solvent was evaporated and the residue was purified by silica gel chromatography $\left(\mathrm{CHCl}_{3}\right)$ to give $96 \mathrm{mg}$ of 17 (53\% recovery), followed by $26.0 \mathrm{mg}$ of 19 ( $32 \%$ based on consumed 17 ) as colorless microcrystals. The product 19 was obtained in $78 \%$ yield under similar reaction conditions at $160^{\circ} \mathrm{C}$ for 6 h. M.p. $210-211^{\circ} \mathrm{C} .{ }^{1} \mathrm{HNMR}\left(\mathrm{CDCl}_{3}\right.$, $500 \mathrm{MHz}) \delta=8.44(\mathrm{~d}, J=0.8 \mathrm{~Hz}, 1 \mathrm{H}), 8.37(\mathrm{~s}, 1 \mathrm{H}), 8.36(\mathrm{~d}, J$ $=0.8 \mathrm{~Hz}, 1 \mathrm{H}), 4.18(\mathrm{tt}, J=12.3,3.7 \mathrm{~Hz}, 1 \mathrm{H}), 2.25(\mathrm{qd}, J=12.3$, $3.7 \mathrm{~Hz}, 2 \mathrm{H}), 1.89(\mathrm{dm}, J=12.3 \mathrm{~Hz}, 2 \mathrm{H}),, 1.77(\mathrm{dm}, J=12.3$ $\mathrm{Hz}, 2 \mathrm{H}), 1.72(\mathrm{dm}, J=12.3 \mathrm{~Hz}, 1 \mathrm{H}), 1.44-1.25$ (m, 3H) ppm; ${ }^{13} \mathrm{CNMR}\left(\mathrm{CDCl}_{3}, 126 \mathrm{MHz}\right) \delta=149.4,149.3,129.9,128.4$, $128.3,119.5,119.1,110.4,109.9,106.0,102.5,56.7,39.4$, 36.3, 35.6 ppm; IR (KBr) $v=2230(\mathrm{w}), 1766$ (s), 1702 (vs) $\mathrm{cm}^{-1}$; UV-vis $\left(\mathrm{CHCl}_{3}\right) \lambda_{\max }=252 \mathrm{sh}(\log \varepsilon=4.70), 258(4.77)$, 281sh (3.83), 324 (3.60), 338 (3.67) nm; MS (70 eV) m/z (\%) $310\left(\mathrm{M}^{+}, 40\right), 267$ (31), 230 (15), 229 (100), 211 (21). HRMS Calcd for $\mathrm{C}_{17} \mathrm{H}_{14} \mathrm{~N}_{2} \mathrm{O}_{2} \mathrm{~S}\left(\mathrm{M}^{+}\right), 310.0776$, found 310.0773 .

\subsection{General Procedure for Mizoroki-Heck Reactions of 19}

A suspension of 19 (1.00 mmol), aryl iodide (1.25 mmol), tetra- $n$-butylammonium bromide $(1.25 \mathrm{mmol}), \mathrm{K}_{2} \mathrm{CO}_{3}(2.50$ $\mathrm{mnol})$, and $\mathrm{Pd}(\mathrm{OAc})_{2}(0.010 \mathrm{mmol})$ in $\mathrm{DMF}(5.0 \mathrm{~mL})$ was heated on an oil bath at $125^{\circ} \mathrm{C}$ for $2 \mathrm{~h}$ under argon. After being cooled to room temperature, the reaction mixture was filtered through a Celite ${ }^{\circledR}$ pad, washed well with ether. The filtrate was poured into water and was extracted with ether $(20 \mathrm{ml} \mathrm{x} \mathrm{3})$. The combined organic layer was washed with a saturated $\mathrm{NaHCO}_{3}$ aqueous solution and brine. After dryness over $\mathrm{Na}_{2} \mathrm{SO}_{4}$, the solvent was evaporated and the residue was purified by silica gel chromatography with $\mathrm{CHCl}_{3}$ as eluant to give the pure product.

20a: Yellowish microcrystals, m.p. $245-246{ }^{\circ} \mathrm{C} .{ }^{1} \mathrm{HNMR}$ $\left(\mathrm{CDCl}_{3}, 400 \mathrm{MHz}\right) \delta=8.33(\mathrm{~s}, 1 \mathrm{H}), 8.22(\mathrm{~s}, 1 \mathrm{H}), 7.87-7.84$ (m, 2H), 7.52-7.49 (m, 3H), 4.11 (tt, $J=12.4,3.6 \mathrm{~Hz}, 1 \mathrm{H})$, $2.18(\mathrm{qd}, J=12.4,3.6 \mathrm{~Hz}, 2 \mathrm{H}), 1.83(\mathrm{dm}, J=12.4 \mathrm{~Hz}, 2 \mathrm{H})$, $1.71(\mathrm{dm}, J=12.4 \mathrm{~Hz}, 2 \mathrm{H}), 1.65(\mathrm{dm}, J=12.4 \mathrm{~Hz}, 1 \mathrm{H}), 1.37-$ $1.21(\mathrm{~m}, J=12.4,3.6 \mathrm{~Hz}, 3 \mathrm{H}) \mathrm{ppm} ;{ }^{13} \mathrm{CNMR}\left(\mathrm{CDCl}_{3}, 100\right.$ MHz) $\delta=167.6,167.6,159.1,143.1,141.8,131.6,130.7$, $130.1,129.8,129.3,128.5,118.1,117.9,114.2,103.3,51.6$, 30.0, 26.2, 25.2 ppm; IR (KBr) $v=2224(\mathrm{~m}), 1769$ (m), 1701 (vs) $\mathrm{cm}^{-1}$; UV-vis $\left(\mathrm{CHCl}_{3}\right) \lambda_{\max }=259 \mathrm{sh}(\log \varepsilon=4.41), 282$ (4.52), 327sh (4.18) nm; MS (70 eV) $\mathrm{m} / z(\%) 386\left(\mathrm{M}^{+}, 72\right)$, 343 (29), 306 (23), 305 (100), 304 (44), 287 (20). HRMS Calcd for $\mathrm{C}_{23} \mathrm{H}_{18} \mathrm{~N}_{2} \mathrm{O}_{2} \mathrm{~S}\left(\mathrm{M}^{+}\right)$, 386.1089, found 386.1090.

20b: Yellowish microcrystals, m.p. $240-242{ }^{\circ} \mathrm{C} .{ }^{1} \mathrm{HNMR}$ $\left(\mathrm{CDCl}_{3}, 500 \mathrm{MHz}\right) \delta=8.33(\mathrm{~d}, J=0.8 \mathrm{~Hz}, 1 \mathrm{H}), 8.22(\mathrm{~d}, J=$ $0.8 \mathrm{~Hz}, 1 \mathrm{H}), 7.89$ (d, $J=8.8 \mathrm{~Hz}, 2 \mathrm{H}), 7.05$ (d, $J=8.8 \mathrm{~Hz}, 2 \mathrm{H})$, 4.15 (tt, $J=12.4,3.6 \mathrm{~Hz}, 1 \mathrm{H}), 3.89$ (s, 3H), 2.23 (qd, $2 \mathrm{H}, J=$ 12.4, $3.6 \mathrm{~Hz}, 1 \mathrm{H}), 1.87$ (d, $J=12.4 \mathrm{~Hz}, 2 \mathrm{H}), 1.75$ (d, $J=12.4$ $\mathrm{Hz}, 2 \mathrm{H}), 1.69$ (d, $J=12.4 \mathrm{~Hz}, 1 \mathrm{H},), 1.42-1.26$ (m, 3H) ppm; ${ }^{13} \mathrm{CNMR}\left(\mathrm{CDCl}_{3}, 126 \mathrm{MHz}\right) \delta=167.756,167.750,162.4$, $159.1,143.3,141.3,130.0,129.0,123.2,117.9,117.5,115.2$, $114.6,101.8,55.7,51.7,30.0,26.2,25.2 \mathrm{ppm} ;{ }^{13} \mathrm{CNMR}$ $\left(\mathrm{CD}_{2} \mathrm{Cl}_{2}, 126 \mathrm{MHz}\right) \delta=167.82,167.79,162.6,159.3,143.5$, $141.7,130.31,130.05,129.3,123.5,118.1,117.4,115.3,114.8$, $102.1,56.0,51.7,30.2,26.5,25.6 \mathrm{ppm}$;[22] IR (KBr) $v=2211$ (w), 1765 (vs), 1706 (s) $\mathrm{cm}^{-1}$; UV-vis $\left(\mathrm{CDCl}_{3}\right) \lambda_{\max }=250$ $(\log \varepsilon=4.45), 257$ (4.49), 294 (4.64), 313 (4.31), 350sh (4.37) nm; MS (70 eV) $m / z(\%) 416\left(\mathrm{M}^{+}, 71\right), 336(14), 335$ (100), 317 (10), 263 (10). HRMS Calcd for $\mathrm{C}_{24} \mathrm{H}_{20} \mathrm{~N}_{2} \mathrm{O}_{3} \mathrm{~S}\left(\mathrm{M}^{+}\right)$, 416.1195, found 416.1191.

20c: Orange microcrystals, m.p. $282-283{ }^{\circ} \mathrm{C} .{ }^{1} \mathrm{HNMR}$ $\left(\mathrm{CDCl}_{3}, 500 \mathrm{MHz}\right) \delta=8.28(\mathrm{~d}, J=0.8 \mathrm{~Hz}, 1 \mathrm{H}), 8.18(\mathrm{~d}, J=$ $0.8 \mathrm{~Hz}, 1 \mathrm{H}), 7.89$ (d, $J=5.5 \mathrm{~Hz}, 2 \mathrm{H}), 6.78(\mathrm{~d}, J=5.5 \mathrm{~Hz}, 2 \mathrm{H})$, $4.16(\mathrm{tt}, J=12.3,3.7 \mathrm{~Hz}, 1 \mathrm{H}), 2.25(\mathrm{qd}, J=12.3,3.7 \mathrm{~Hz}, 2 \mathrm{H})$, $1.89(\mathrm{dm}, J=12.3 \mathrm{~Hz}, 2 \mathrm{H}), 1.77(\mathrm{dm}, J=12.3 \mathrm{~Hz}, 2 \mathrm{H}), 1.71$ $(\mathrm{dm}, J=12.3 \mathrm{~Hz}, 1 \mathrm{H}), 1.43-1.28(\mathrm{~m}, 3 \mathrm{H}) \mathrm{ppm} ;{ }^{13} \mathrm{CNMR}$ $\left(\mathrm{CDCl}_{3}, 126 \mathrm{MHz}\right) \delta=167.7,167.7,160.0,152.2,143.8$, $140.5,129.7,129.4,128.1,117.8,117.7,116.7,115.2,111.9$, 98.9, 51.2, 40.1, 29.9, 26.0, 25.1 ppm; IR (KBr) $v=2212(\mathrm{w})$, 1760 (s), 1704 (s), 1366 (vs) cm ${ }^{-1}$; UV-vis $\left(\mathrm{CH}_{3} \mathrm{CN}\right) \lambda_{\max }=$ 256sh ( $\log \varepsilon=4.57), 266$ (4.63), 310 (4.07), 328 (4.11), 414 (4.58) nm; MS (70 eV) m/z (\%) 430 (29), $429\left(\mathrm{M}^{+}, 100\right), 348$ (10), 347 (33), 346 (20). HRMS Calcd for $\mathrm{C}_{25} \mathrm{H}_{23} \mathrm{~N}_{23} \mathrm{O}_{2} \mathrm{~S}\left(\mathrm{M}^{+}\right)$, 429.1511 , found 429.1518 .

\section{Conclusion}

We have demonstrated that the novel benzo[b]thiophene-5,6-dicarboximides 6,9 , and 10 can be synthesized easily by phosphine-assisted annulation between thiophene-2,3-dicarbaldehydes and various $N$-substituted maleimides. Introduction of a cyano group to the thiophene moiety of the $N$-cyclohexyl product was achieved by copper-mediated coupling reaction via its bromo derivatives and Mirozoroki-Heck reaction of the 3-cyano product led to 2-aryl-3-cyano derivatives. Although the emission quantum yields of 6 are low, 2-aryl-3-cyano derivatives 20 indicate the enhanced emission quantum yield up to $27 \%$. Also, we have demonstrated that the absorption and emission wavelengths are tunable by a substituent on the aryl moiety in this structure of 2-aryl-3-cyanobenzo[b]thiophene-5, 6-dicarboximide. 


\section{References}

[1] P. Nandhikonda and M. D. Heagy, "Dual fluorescent $N$-aryl-2,3-naphthalimides: applications in ratiometric DNA detection and white organic light-emitting devices", Org. Lett. 2010, 12, 4796-4799.

[2] X. Zhan, A. Facchetti, S. Barlow, T. J. Marks, M. A. Ratner, M. R. Wasielewski, and S. R. Marde, "Rylene and diimides for organic electronics", Adv. Mater. 2011, 23, 268-284.

[3] P. Nandhikonda and M. D. Heagy, "An abiotic fluorescent probe for cardiac troponin l”, J. Am. Chem. Soc., 2011, 133, 14972-14974.

[4] K. Hutt, R. Hernadez, and M. D. Heagy, "Toward intrinsically fluorescent proteomimetics: Fluorescent probe response to alpha helix structure of poly- -benzyl-L-glutamate", Tetrahedron Lett. 2006, 16, 5436-5438.

[5] N. Sakai, J. Mareda, E. Vauthey, and S. Matile, "Core-substituted naphthalenediimides", Chem. Commun. 2010, 46, 4225-4237.

[6] Y. Zhou, L. Ding, K. Shi, Y.-Z. Dai, N. Ai, J. Wang, and J. Pei, "A non-fullerene small bulk heterojunction solar cells", Adv. Mater. 2012, 24, 957-961.

[7] T. V. Pho, F. M. Toma, M. L. Chabinyc, and F. Wudl, "Self-assembling decacyclene triimides prepared through a regioselective hextuple Freidel-Crafts carbamylation", Angew. Chem. Int. Ed. 2013, 52, 1446-1451.

[8] Y. Zhong, B. Kumar, S. Oh, M. T. Trinh, Y. Wu, K. Elbert, P. Li, X. Zhu, S. Xiao, F. Ng, M. L. Steigerwald, and C. Nuckolls, "Helical ribbons for molecular electronics", J. Am. Chem. Soc. 2014, 136, 8122-8130.

[9] M. Oda, H. Shimosasa, Y. Kumai, A. Ohta, and R. Miyatake, "An improved synthesis of arenedicarboximides by phosphine-assisted annulation of arene-1,2-dicarbaldehyde with $N$-substituted maleimide", Modern Chem. 2014, 2 (4), 29 35 .

[10] H. Shimosasa, R. Miyatake, N. Kobayashi, and M. Oda "Synthesis and emission behavior of 1,3-diarylisobenzofuran5,6-dicarboximides and their transformation into naphthalene-2,3:6,7-bis(diacboximide)s", Modern Chem. 2016, $4(6), 16-23$.
[11] T. Yanagisawa, N. Kobayashi, H. Shimosasa, Y. Kumai, R. Miyatake, and M. Oda, "Synthesis and fluorescence property of 2,3-naphthalimie deriveatives bearing phenyl substituents on the naphthalene skeleton", Dyes Pigments in press, http://dx.doi.org/10.1016/j.dyepig.2016.09.050.

[12] M. J. Haddadin, B. J. Agha, and R. F. Tabri, "Syntheses of some furans and naphtho[2,3-c] derivatives of furan, pyrrole, and thiophene", J. Org. Chem. 1979, 44, 494-497.

[13] S.-M. Yang and J.-M. Fang, "Coupling reactions and coupling-alkylations of thiophenecarbaldehydes promoted by samarium diiodide", J. Org. Chem. 1999, 64, 394-399.

[14] P. Valat, V. Wintgens, J. Kossanyi, L. Biczók, A. Demeter, and T. Bérces, "Influence of geometry on the emitting properties of 2,3-naphthalimides", J. Am. Chem. Soc. 1992, 114, 946-953.

[15] For examples, an emission quantum yield of $N$-ethylnaphthlene-2,3-dicarboximide, corresponding to $6 \mathrm{~b}$, in chloroform is 0.37 and that of $N$-phenylnaphthalene2,3-dicarboximide, corresponding to $6 \mathrm{~d}$, is 0.10 .

[16] The low quantum yields of $6 \mathrm{e}$ and $6 \mathrm{f}$ are ascribed also to a heavy atom effect of the bromine and iodine atoms.

[17] J. F. D. Chabert, L. Joucla, E. David and M. Lemaire, "An efficient phosphine-free palladium coupling for the synthesis of new 2-arylbenzo[b] thiophenes", Tetrahedron 2004, 60, 32213230 .

[18] Although Lemaire et al. reported cyanation of 3-bromobenzo $[b]$ thiophene with $\mathrm{CuCN}$ in $N, N$-dimthylacetamide in ref 9 , cyanation of 12 under the similar conditions yielded 13 in low yield.

[19] M. P. Cava, A. A. Deana, K. Muth, and M. J. Mitchell, "N-Phenylmaleimide", Org. Synth. Coll. Vol. 5, 1973, 944-946.

[20] R. Tona and R. Häner, "Crosslinking of diene-modified DNA with bis-maleimides", Mol. BioSyst. 2005, 1, 93-98.

[21] H. Shen, K. C. Vollhardt, and C Peter, "Remarkable switch in the regiochemistry of the iodination of anilines by $\mathrm{N}$-iodosuccinimide: synthesis of 1,2-dichloro-3,4-diiodobenzene", Synlett 2012, 23, 208-214.

[22] Although the ${ }^{13} \mathrm{C}$ NMR spectrum of $20 \mathrm{~b}$ in $\mathrm{CDCl}_{3}$ shows only 19 signals, which are short for the structure of $20 \mathrm{~b}$, the spectrum in $\mathrm{CD}_{2} \mathrm{Cl}_{2}$ indicates 20 signals enough for the structure of $20 \mathrm{~b}$. 